\section{Neue Strömungen in der Denkmalpflege}

Vor kurzem sind zwei Schriften über moderne Denkmalpflege erschienen, deren Verfasser, jeder in seinem Fache, ein wohlbegründetes Ansehen genießen, und namentlich auch auf dem Gebiete, dem ihre jüngsten Enunziationen gelten, den unbestrittenen Anspruch erheben dürfen, mit Ernst und Aufmerksamkeit gehört zu werden. Eine von diesen Schriften ist die Wiedergabe einer akademischen Rede, welche der ordentliche Professor der Kunstgeschichte an der Straßburger Universität Dr. Georg GottFried Dehio gelegentlich der diesjährigen Geburtstagsfeier des deutschen Kaisers gehalten hat. ${ }^{1}$ ) Die andere hat den Berliner Architekten Bodo EBHARdT zum Verfasser, der sich in Deutschland namentlich durch zahlreiche Restaurierungen mittelalterlicher Burgen einen Namen zu erwerben wußte; die Ausführungen in seiner jüngsten Broschüre ${ }^{2}$ ) behandeln auch ausschließlich die Pflege älterer Burgbauten, und wenn der Titel gleichwohl ein umfassenderes Thema anzukündigen scheint, so läßt sich diese Erweiterung nur insofern rechtfertigen, als bei dem engen Zusammenhange, der zwischen allen Denkmalgebieten untereinander in bezug auf ihre Pflege herrscht, von der Behandlung von Burgen in der Tat die Gedanken des Verfassers über eine analoge Behandlung anderer alter Bauwerke mit logischer Notwendigkeit abgeleitet werden können.

Was dem gleichzeitigen Erscheinen dieser beiden Schriften im gegenwärtigen Momente einen besonderen Wert verleiht, ist der Umstand, daß ihre vergleichende Lektüre einen tiefen Einblick gewährt in die Wandlung, die sich heute (und bereits seit Jahren) in der grundsätzlichen Auffassung von der innersten Triebfeder und den

1) Denkmalschutz und Denkmalpflege im neunzehnten Jahrhundert. Rede zur Feier des Geburtstages Sr. Maj. des Kaisers, gehalten in der Aula der Kaiser Wilhelm-Universität am 27. Januar-1905 von Dr. GEorg GotTFried Dehio, o. Prof, der Kunstgeschichte. Straßburg J. H. Ev. Heitz $19058^{0} 27 \mathrm{~S}$.

$\left.{ }^{2}\right)$ Über Verfall, Erhaltung und Wiederherstellung von Baudenkmalen mit Regeln für praktische Ausführungen. Von Bodo EBHardt, Architekt, Berlin Franz EBHARDT \& Co. $19058^{0} 49 \mathrm{~S}$. dadurch bedingten äußeren Aufgaben der Denkmalpflege vollzieht, und zugleich in die Hindernisse, die einem glatten und normalen Verlaufe dieser Wandlung entgegenstehen, und denen auch die zahlreichen oft unüberbrückbar scheinenden Mißverständnisse und Meinungsverschiedenheiten in den einzelnen Restaurierungsfragen zum wesentlichen Teile zugeschrieben werden müssen. Um einer von uns für nützlich und dringend empfundenen Klarstellung dieser Verhältnisse willen, und nicht aus Absicht einer einfachen literarischen Anzeige, derengleichen vom Programme dieser "Mitteilungen" ohnehin ausgeschlossen sind, soll im folgenden auf gewisse Grundgedanken über das Wesen der Denkmalpflege, die in den genannten zwei Schriften zum Ausdrucke gebracht erscheinen, kritisch eingegangen werden. Es ist selbstverständlich, daß auf dem Gebiete der Denkmalpflege so erfahrene Männer auch im einzelnen eine Menge Dinge vorzubringen wußten, die dem Denkmalfreunde wie dem berufsmäßigen Denkmalpfleger Nutzen und Anregung gewähren; an dieser Stelle darauf einzugehen, lag jedoch nicht in unserer Absicht.

Daß der Standpunkt, den der Kunsthistoriker DE़нIo zur modernen Denkmalpflege einnimmt, demjenigen des ausübenden Künstlers EBHARDT vielfach diametral entgegengesetzt ist, dünkt uns heute fast selbstverständlich; zahllose polemische Äußerungen aus beiden Lagern in der Literatur und auf den alljährlich wiederkehrenden Denkmaltagen haben uns längst daran gewöhnt, die Stellung der schaffenslustigen Architekten einerseits, der jedem Eingriffe in die Denkmäler grundsätzlich widerstrebenden Kunsthistoriker anderseits, als zwei Extreme zu betrachten, zwischen denen eine zwar pietätvolle, aber den unwiderstehlichen Mächten der realen Wirklichkeit klug Rechnung tragende Denkmalpflege jeweils die richtige Mitte $\mathrm{zu}$ treffen hat. Freilich läßt sich angesichts des Umstandes, daß doch beide Parteien ein und dasselbe Ziel im Auge haben, schon von vornherein der Gedanke nicht unterdrücken, daß bei gutem Willen zwischen den Extremen wenigstens eine Annäherung gefunden werden könnte, die der Sache gewiß nur zum Vorteil gereichen würde. Bevor wir aber diesem Gedanken nachgehen, wollen wir uns des Zufalles, der uns gleichzeitig Äußerungen 
aus beiden gegnerischen Lagern beschert hat, noch aus dem Grunde freuen, weil wir von vornherein hoffen dürfen, in der Äußerung des einen sofort die Kritik der Äußerungen des andern zu finden und dadurch unserer eigenen Kritik Vorschub geleistet zu sehen.

Deнiо - um mit diesem zu beginnen gibt über die Haupt- und Grundfrage aller Denkmalpflege zwar kurze, aber sehr bestimmte und sorgfältiger Erwägung würdige Erklärungen. "Wir konservieren ein Denkmal nicht weil es schön ist, sondern weil es ein Stück unseres nationalen Daseins bildet." "Denkmale schützen heißt nicht Genuß suchen, sondern Pietät üben." „Ästhetische und selbst kunsthistorische Urteile schwanken; hier (d. h. in dem "Stück nationalen Daseins") ist ein unveränderliches Wertkennzeichen gefunden."

Das sind allerdings Sätze von programmatischer Bedeutung, in denen die Wandlung in der Auffassung vom Wesen der Denkmalpflege zum schärfsten Ausdrucke gelangt. Deнго empfindet, daß das "künstlerische und historische Interesse", womit man im XIX. Jh. den Denkmalbegriff definiert hat, heute diesen Begriff nicht mehr erschöpft. Weder das egoistische Lustgefühl, das durch Form und Farbe des Denkmals erzeugt wird, noch die verstandesmäßige Befriedigung über die durch das Denkmal in uns angeregten historischen, namentlich kunsthistorischen Ideenassoziationen reichen aus, um die oft schwärmerische Begeisterung des modernen Menschen für die "Denkmale" zu erklären, zumal beiden genannten Wertursachen sowohl dem Kunstgeschmacke als dem historischen Interesse - jeweilen bloß relative Gültigkeit zukommt. Deнio empfindet ganz richtig, daß die ästhetisch-wissenschaftliche Schablone der „kunstund historischen Denkmale" heute nicht mehr paßt, und daß das eigentlich zwingende Motiv des Denkmalkultus in einem altruistischen Gefühle beruht, das uns Pietät, das heißt Aufopferung gewisser entgegenstehender egoistischer Bestrebungen als innere Pflicht auferlegt. Dieses altruistische Gefühl faßt aber Deнio als ein nationales: "Wir schützen das Denkmal als ein Stück nationalen Daseins."

Diese Fassung dünkt uns, um es gleich zu sagen, eine zu enge; Deнio steht damit offenbar doch noch unter der Nachwirkung des Bannes der Anschauung des XIX. Jh., welche die Bedeutung des Denkmals wesentlich im "historischen“ Momente gesucht hatte.

In welchem Sinne seine Definition des Denkmals als "Stück nationalen Daseins" zu verstehen ist, erläutert Dенго selbst an einem Beispiele, indem er den Verlust des Jamnitzer Pokals an das Louvremuseum beklagt. Ein Denkmal deutschen Kunstschaffens müsse nun der Deutsche in Paris aufsuchen! Aus solchen Äußerungen spricht der gleiche Stolz auf die nationale Leistung, wie aus der französischen Definition der "Nationaldenkmäler", welche einen Teil der Gloire der französischen Nátion ausmachen. Das altruistische Moment in diesem Gefühle ist offenbar ein beschränktes: es wird sofort ein egoistisches, sobald es mit Bezug auf Angehörige einer andern Nation empfunden wird. Es heißt dann Ruhmsucht, und nicht mehr Pietät. Haben wir aber nicht unzählige Male im Anblick eines Denkmals geschwelgt, ohne uns auch nur im leisesten seines nationalen Ursprungs bewußt $\mathrm{zu}$ werden? Und haben uns andere Denkmäler darum weniger Wohlgefallen eingeflößt, weil sie einem ausländischen, etwa dem italienischen Kunstschaffen entstammten? Hieraus erheben sich Bedenken gegen die Gemeingültigkeit der Demioschen Definition. Trachten wir uns an der Hand von Beispielen darüber klar zu werden.

Kürzlich wurde durch die Nachricht, daß dem geplanten Eisenbahnbau in der Wachau einige alte Häuser, namentlich im Orte Weißenkirchen, zum Opfer fallen sollen, manchem Denkmalfreunde aufrichtiges Leid verursacht. Frug sich aber der also Betroffene, was ihm mit diesen Häusern verloren ging, so begegnete er überall tröstlichen Erwägungen, soweit bloß das „künstlerische und historische Interesse" in Betracht kam. Die zu hohen oder $\mathrm{zu}$ schmalen Verhältnisse, die auf rohen Bogen unregelmäßig vorgewölbten Obergeschosse, die derben Säulen, winkeligen Freitreppen usw. würde man an jedem modernen Bau von künstlerischem Standpunkte unerträglich finden; in kunsthistorischer Beziehung besitzen wir dafür anderwärts zahlreiche, ungleich wertvollere Ersatzstücke; historische Erinnerungen endlich, die sonst in der Wachau von 
Richard Löwenherz bis auf Napoleon zahlreich genug entgegentreten, fehlen gerade jenen zum Abbruche bestimmten Häusern gänzlich. Was ist es also, dessen drohender Verlust uns trotz aller Vernunfterwägungen ein untilgbares Leid empfinden läßt? Es kann nichts anderes sein, als das "Alte" an und für sich, das Nichtmoderne, das Zeugnis eines Schaffens früherer menschlicher Generationen, deren Nachfahren wir selbst bilden. So wie wir unsere Vorfahren gleichsam als eine Verlängerung unseres eigenen Daseins nach rückwärts in der Zeit auffassen dürfen, stellen sich uns entsprechenderweise auch die Denkmäler als eine Verlängerung unseres eigenen Schaffens nach rückwärts dar, und in diesem Lichte betrachtet, gewinnen sie für uns ein solches Interesse, daß wir ihrer Erhaltung selbst Opfer an modernen, zeitlichen Gütern bringen.

Solchermaßen erscheinen uns die alten Häuser von Weißenkirchen in der Tat als ein Stück unseres eigenen Daseins, und insofern als sie einstmals von Deutschösterreichern gebaut wurden und wir uns während ihrer Betrachtung als Deutschösterreicher fühlen, auch als ein Stück unseres nationalen Daseins. Aber wenn man selbst davon absieht, daß die Wiege so manches also Empfindenden fern von der Wachau gestanden war, und seine Vorfahren sich nicht immer zur deutschösterreichischen Nationalität bekannt haben, vermögen gewiß viele das Bewußtsein nicht zu unterdrücken, daß ihnen etwa dieLauben in Trient oder die aus ganz anderen Kulturverhältnissen entstandenen Gäßchen im Palastviertel von Spalato genau das gleiche Gefühl der unbedingten Lust an der Anschauung des Alten an und für sich einzuflößen pflegen als die Wachauer Häuser. Und warum - wenn es gestattet ist ein Exempel aus der eigenen Erfahrung vorzubringen -- habe ich es von Júgendjahren an stets instinktiv vermieden, zu Rom im modernen Fremdenviertel mein Quartier aufzuschlagen und habe dafür stets die als fieberig verrufene Tiberstadt aufgesucht, deren Gassen wenigstens das unverfälschte, wenn auch einfache Gepräge der Barockzeit zur Schau tragen? Und das selbst schon zu Zeiten, als ich noch als gelehriger Schüler meiner Lehrmeister im Barockstil als solchem die abscheulichste Verirrung des menschlichen Kunstgeistes erblicken zu müssen glaubte! In welch anderem konnte da der Denkmalwert dieser von mir wider die verstandesmäßige Erwägung vorgezogenen Menschenwerke beruhen, als in ihrem Alterswert an und für sich, ganz abgesehen von der Nationalität ihrer Errichter? In diesem Lichte betrachtet, erscheinen sie uns gewiß auch als ein Stück unseres Daseins, aber nicht des nationalen, sondern des menschlichen Daseins. Der Nationalegoismus erscheint damit zu einem Menschheitsegoismus abgeschwächt, das der Denkmalpflege zugrunde liegende Gefüh1 einem rein altruistischen wesentlich nähergebracht.

Als ein Stück seines nationalen Daseins, wie Deнio will, können die Denkmale somit streng genommen nur demjenigen gelten, der keine anderen Denkmale kennt, als jene seines Heimatslandes, was aber heute wohl nurmehr bei wenigen Gebildeten zutrifft. Ferner darf man zugeben, daß bei zahlreichen Denkmalfreunden der Heimatssinn so außerordentlich einseitig entwickelt ist, daß die heimatlichen Denkmale in ihm weit rascher und intensiver die Stimmungsgefühle erwecken, als solche die auf fremdem Boden erwachsen sind. Aber da handelt es sich bloß um sekundäre Unterschiede: das Hauptmotiv des Denkmalkultus auf dieser Entwicklungsstufe (die, wie gleich gezeigt werden soll, heute auch bereits überschritten ist) bleibt das „Menschheitsgefühl“, mag es auch gelegentlich überwiegend bloß den Menschen von einer bestimmten Nationalität gelten, d. h. in der beschränkten Form des Nationalgefühls zum Ausdrucke gelangen.

Die letzten Ziele des modernen Denkmalkultus werden aber erst dann völlig klar, wenn man auch den wachsenden Sinn für die Pflege der "Naturdenkmale" mit in Betrachtung zieht, was Deнio allerdings - für seinen Standpunkt sehr bezeichnenderweise - unterlassen hat. Eine Dorflinde mag man am Ende noch als ein Stück nationalen Daseins ansehen, weil sie von unseren Vorfahren gepflanzt wurde; aber ein gigantischer wilder Waldbaum oder eine senkrecht gewachsene Felswand ist von der Natur selbsttätig hervorgebracht, ohne Zutun menschlicher Hände. Warum empfinden wir es dann als einen Frevel, die Hand daran zu legen, den Baum zu fällen, die Felswand zu sprengen, und ihnen damit gleichsam 
das Lebenslicht auszublasen? Warum reklamieren wir auch für diese Naturerzeugnisse das Recht, sich gleichsam ungestört ausleben zu dürfen? Wir achten eben auch in ihnen die Zeugnisse vergangenen Daseins, Lebens und Schaffens, aber allerdings nicht des Daseins der Nation und auch nicht des Daseins der Menschheit, wie auf der vorher fixierten Entwicklungsstufe überhaupt, sondern des Daseins der Natur. Im Kultus der „Naturdenkmale" ist auch der letzte Rest von Egoismus der auf die Menschheit bezügliche - überwunden, und mit der Teilnahme an den vergangenen Geschicken der außermenschlichen Natur der volle Altruismus erreicht. Der Kultus der Naturdenkmale ist der alleruninteressierteste: er verlangt von uns Lebenden mitunter Opfer für ein lebloses Naturding.

So sehen wir den modernen Denkmalkultus immer mehr dahin drängen, das Den kma 1 nicht als Menschenwerk, sondern als Naturwerk zu betrachten, und so erklärt sich auch die schon öfter gemachte Beobachtung, daß ihm von den Gebieten der modernen Kunst keine näher steht, als die Landschaftsmalerei. Tierschutzbestrebungen und Denkmalschutzbestrebungen sind im Grunde aus einer und derselben Wurzel hervorgegangen. Bei beiden ist der Wunsch maßgebend, etwas zu vermeiden, was unser subjektives Gefüh1 verletzt, nicht bloß unser Kollektivgefühl für Menschenwürde oder gar bloß für Nationalwürde. Wir empfinden es als ein rein persönliches Leid, daß die Weißenkirchner alten Häuser fallen sollen: nicht deshalb, weil sie von unseren nationalen Vorfahren erbaut und bewohnt waren, und auch nicht so sehr, weil sie darüber hinaus Zeugnisse unserer eigenen menschheitlichen Vergangenheit darstellen, sondern weil sie überhaupt etwas in früheren Zeiten Gewordenes von bestimmtem individuellem Charakter sind und damit ein Recht darauf erworben haben, sich womöglich nach ihren eigenen Erhaltungsbedingungen auszuleben.

Wiewohl nun nach dem Gesagten DeHIos Zurückführung des modernen Denkmalkultus auf ein Bedürfnis des Nationalgefühls den Sachverhalt keineswegs erschöpft, hat doch die darin enthaltene Grunderkenntnis, daß es sich dabei um die Pflege eines Gefühls handelt, bereits genügt, um DEHIO die sozialistische Tendenz des modernen Denkmalkultus zu erschließen. „Das Interesse, das die Gesamtheit an einem Denkmale hat, überwiegt ganz unermeßlich das Interesse des Individuums." Das soll soviel heißen, als daß in der Regel bloß der einzelne Besitzer des Denkmals selbst von seinem egoistischen Standpunkte ein Interesse an der Beseitigung eines Denkmals haben kann, weil ihm seine Erhaltung solche materielle Opfer auferlegt oder ihn an der Erreichung anderer so gewichtiger Vorteile hindert, daß durch dieses starke egoistische Interesse sein altruistisches, die Erhaltung forderndes übertäubt und erstickt wird. Dagegen haben alle übrigen Menschen bloß das altruistische Interesse an der Erhaltung des Denkmals, worin sie durch keinerlei konkurrierende egoistische Interessen beirrt und behindert werden. Es ist dann gewiß nur billig zu verlangen, daß der Wunsch so vieler, wie sie die Gesamtheit ausmachen, gegenüber dem entgegengesetzten Wunsche eines einzelnen Berücksichtigung finde; dadurch gewinnt aber die Forderung nach öffentlichem Denkmalschutz allerdings einen sozialistischen Charakter. Eines ist jedoch dabei unumgängliche Voraussetzung: daß in der Tat die Gesamtheit oder doch wenigstens ein sehr wesentlicher und maßgebender Teil derselben die Forderung auf Erhaltung des Denkmals erhebt. Alle Schutzparagraphen würden sich ohnmächtig erweisen, wenn nicht das "Volk" von der Notwendigkeit des Schutzes überzeugt wäre und seine Ausübung selbst in die Hand nähme. Um aber diese Notwendigkeit als eine zwingende zu empfinden, genügt weder eine Auffassung, die im Denkmal bloß ein ästhetisches Lustmittel erkennt, noch jene andere, die damit wissenschaftlich-historische Liebhabereien befriedigen will. Der Denkmalwert muß vielmehr ein Gefühlswert und damit zur Gefüh1ssache der breiten Masse, wenigstens der Gebildeten, geworden sein, und das kann überhaupt erst von dem Momente an zutreffen, als wir im Denkmal ein „Stück des eigenen Daseins" zu schätzen gelernt haben.

Dies ist aber bloß eine, und zwar gewissermaßen die subjektive Seite, in welcher die sozialistische Tendenz in den modernen Denkmalschutzbestrebungen zutage tritt. Dazu gesellt sich noch 
eine andere objektive Seite, die in der Demokratisierung des Denkmals selbst beruht. Die Definition der „kunst- und historischen Denkmale" hatte noch eine aristokratische Auslese bedingt; diese muß aber in Wegfall kommen, sobald es sich beim Denkmal wesentlich nurmehr um das Altsein als solches handelt. Es wird vom Denkmal nichts mehr gefordert, als deutliche Altersspuren und eine hinreichende individuelle Geschlossenheit, wodurch es sich seiner Umgebung und der ganzen übrigen Welt entgegensetzt.

Daß dieser Sachverhalt heute noch vielfach verkannt, der Denkmalwert noch immer vorwiegend im "Schönen" und im "Historischen" gesucht wird, ist die eigentliche Quelle aller Unklarheiten, Mißverständnisse und erbitterten Streitigkeiten auf diesem Gebiete. Noch immer werden vielfach selbst solche, die angesichts jener alten Häuser der Wachau die Gefühìswirkung der Stimmung deutlich erfahren, sich trotzdem damit abmühen, zu erweisen, wie man im XVI. und XVII. Jh. "schöner" gebaut habe als heutzutage. Und die Aufgeklärteren werden betonen, daß diese Häuser kunsthistorische Details zeigen, die für die Architektur der Renaissanceund Barockzeit charakteristisch sind, und daß sie zugleich auch von der geschichtlichen Entwicklung auf zahlreichen anderen Gebieten menschlicher Kultur in den deutschösterreichischen Erblanden Zeugnis ablegen. Diese beiden Momente kommen auf der heutigen Stufe unserer Kulturentwicklung nebenher zweifellos auch noch mehr oder minder ansehnlich in Betracht; aber das erstere, soweit es überhaupt ernst empfunden wird und nicht auf Selbsttäuschung beruht, ist nur dem ästhetisch Gebildeten, das zweite bloß dem wissenschaftlichhistorisch Gebildeten zugänglich. Das Entscheidende in der Wirkung eines Denkmals auf den modernen Beschauer bildet keines von beiden. Dieses liegt vielmehr in einem an sich undefinierbaren Gefühl, das sich lediglich als eine unstillbare Sehnsucht nach Anschauung eines "Alten" äußert. Wir bemerken an dem Hause, daß es "alt" ist, und sind darüber an und für sich entzückt. Daß man diesen Sachverhalt so lange verkannt hat und ihn sich selbst heute noch vielfach mit allen Kräften zu verhehlen trachtet, kann wohl am verständlichsten aus dem Unbehagen erklärt werden, das der moderne Gebildete jedesmal empfindet, wenn er einem nicht sofort verstandesmäßig zu Fassenden gegenübertritt. Der Beschauer will sich nicht eingestehen, daß er das Gefüh1, das er angesichts eines Denkmals empfindet, sich nicht sofort erklären, könne, und so täuscht er sich vor, das Denkmal gefalle ihm, weil es schön oder weil es historisch interessant wäre. Auf diesem Wege ist wohl auch DeHio, trotz seiner Erkenntnis, daß der Denkmalwert auf einem Gefühle beruht, noch dazu gelangt, das Nationalbewußtsein als die entscheidende Triebfeder des Denkmalkultus hinzustellen, weil dieses Bewußtsein, auf der Rassezusammengehörigkeit begründet, einen wenn auch noch nicht physikalisch erklärten, so doch als tatsächlich vorhanden allgemein anerkannten Faktor bildet. Daß eine solche Auslese der Denkmale nach dem Gesichtspunkte ihrer Wichtigkeit für unsere nationale Vergangenheit, wie schon früher angedeutet wurde, noch eine Nachwirkung des Bannes der im XIX. Jh. herrschend gewesenen "historischen" Auffassung vom Wesen des Denk mals verrät und hiedurch eine Brücke zu dieser früheren, heute noch in weitverbreitetem Ansehen stehenden Auffassung bildet, mochte für Dвніо ebenfalls etwas Lockendes und zugleich Beruhi. gendes gehabt haben. Nach unseren früheren Ausführungen wird man aber, wenn die letzten Ziele der modernen Denkmalpflege genannt werden sollen, DeHios Nationalgefühl mindestens durch das Menschheitsgefühl, wo nicht (wozu der Kultus der Naturdenkmale heute schon zwingt) durch ein Daseinsgefühl überhaupt ersetzen müssen. Die Denkmale entzücken uns hienach als Zeugnisse dafür, daß der große Zusammenhang, von dem wir selbst einen Teil bilden, schon lange vor uns gelebt und geschaffen hat. Eine solche Erklärung bedeutet ebensowenig einen Sprung ins Transzendente, als jene Denios, die auf dem Nationalbewußtsein basiert.

Aber selbst wenn man die im vorstehenden gegebene Analyse und Erklärung des Gefühls, das man angesichts eines Denkmals empfindet, nicht zutreffend finden sollte: das eine wird man sich endlich klar und deutlich eingestehen müssen, daß es ein unwiderstehlich zwingendes Gefühl ist, das uns zum Denkmalkultus treibt, und nicht ästhe. tische und historische Liebhabereien. Wären es 
nur die letzteren, dann wäre nichts ungerechtfertigter, als der Ruf nach gesetzlichem Schutz. Wie könnten sich die Ästheten und Wissenschaftler herausnehmen zu verlangen, daß um ihrer künstlerischen und historischen Liebhabereien willen das Privatrecht auf tausend Punkten durchbrochen, die freie Verfügung nach vernunftgemäßen Rücksichten über die Denkmale eingeschränkt werde? Nur auf dem Vorhandensein und der allgemeinen Verbreitung eines Gefühls, das, verwandt dem religiösen Gefühle, von jeder ästhetischen oder historischen Spezialbildung unabhängig, Vernunfterwägungen unzugänglich, seine Nichtbefriedigung einfach als unerträglich empfinden läßt, wird man mit Aussicht auf Erfolg ein Denkmalschutzgesetz begründen können.

Das trotz vorgeschrittener Einsicht immer noch Halbe und Unzulängliche in der Auffassung Deнios vom „Denkmal als einem Stück unseres nationalen Daseins" mußte sich naturgemäß namentlich dann rächen, sobald der Versuch gemacht wurde, daraus die Grundsätze für die praktische Behandlung der Denkmale abzuleiten. "Konservieren, nicht restaurieren" lautet Denios kurze Formel dafür, und die Restauratoren kommen in seinen Ausführungen übel genug weg. Diesen entschiedenen Einspruch gegen alle Wiederherstellungen an Denkmalen hat Deнio schon vor zwei Jahren auf dem deutschen Denkmaltage vertreten. Daß er damit auf den heftigsten Widerspruch der restaurierenden Architekten gestoßen ist, läßt sich unschwer begreifen. Schwerer verständlich ist es jedoch, daß ihm von Seite seiner kunsthistorischen Kollegen nichtso eifrig sekundiert wurde, als er vielleicht selbst erwartet haben mochte. Es scheint, daß die Kunsthistoriker in Demıs Polemik eine schwache Seite merkten und daher trotz der Zustimmung, die sie den durch ihn proklamierten Grundsätzen entgegenbringen mußten, sich in ihren bezüglichen Erklärungen Reserve auferlegten. Die schwache Seite ist in der Tat vorhanden; sie beruht in nichts anderem als jenem wiederholt betonten Reste von „nationalhistorischer" Bedeutung, den Deнio mit dem Denkmal neben oder trotz der auch von ihm erkannten Gefühlsbedeutung desselben noch immerverknüpfen möchte. Der Nachweis hiefür, der zugleich über die schwersten der zwischen restaurierenden Architekten und Kunsthistorikern vorhandenen Mißver- ständnisse Aufklärung bietet, soll später geführt werden; um die nötige klare Basis dafür zu schaffen, empfiehlt es sich zunächst, die Anschauungen eines modernen ausübenden Künstlers über Wesen und Behandlungsgrundsätze der Denkmalpflege kennen zu lernen.

Der Architekt BoDo EBHARDT gibt zwar in seiner Schrift keine präzise Definition des „Denkmals", aber er läßt uns nicht im geringsten in Zweifel darüber, daß für ihn der Wert eines Denkmals wesentlich in seiner historischen Bedeutung ruht. Nach EBHARdTs Auffassung müßte die Definition etwa lauten: Wir schützen ein Denkmal, weil es uns ein in seiner Art geschlossenes Bild von einer früheren kulturgeschichtlichen Entwicklungsstufe gewährt. Was uns etwa angesichts einer Burg aus dem XIII. Jh. mit einem Lustgefühl erfüllt, wären hienach hauptsächlich die Ideenassoziationen gelehrter Art, die sich daran knüpfen; wir freuen uns, an der Burg alle jene Dinge wiederzufinden, die das uns auf dem Wege gelehrter Bildung bekannt gewordene Kulturleben des XIII. Jh. charakterisieren. Ein Lustgefüh1 ist es also auch nach dieser Auffassung, das wir durch das Denkmal in uns erregt zu erhalten erwarten; aber dieses Gefühl ist kein so unmittelbares, wie das ästhetische, das durch Auffassung, Form und Farbe des Denkmals bedingt ist, oder vollends jenes hinreißende Stimmungsgefüh1, das durch den Anblick des "Alten" an und für sich in uns hervorgerufen wird, sondern es gelangt erst auf dem Umwege bewußter Reflexion, der Verknüpfung äußerlich angelernter Ideen zustande.

Das kulturgeschichtliche Bild, dessen Hervorrufung wir gemäß dieser Auffassung vom Denkmal verlangen, ist nun ein um so deutlicheres, die Ideenassoziationen, durch die dieses Bild hervorgerufen wird, sind um so reicher, je vollständiger das Denkmal erhalten ist. Eine vollkommen erhaltene Burg ist daher vom Standpunkte dieser Auffassung einer Burgruine entschieden vorzuziehen. Wie aber, wenn eben bloß eine Ruine vorhanden ist, die uns nur mangelhaffte Anhaltspunkte für die Anknüpfung der nötigen Ideenassoziationen gewährt? Um diese Ruine für den Denkmalkultus nach EBHARDTs Auffassung vollwertig zu machen, müssen die mangelnden Glieder nach Tunlichkeit ergänzt, die Lücken ausgefüllt 
werden: mit einem Worte, die in Ruinen gesunkene Burg muß wieder hergestellt werden. Nur eins ist dabei strengste Bedingung: Das Ergänzte muß genau die Formen nachahmen, die an der gleichen Stelle früher vorhanden waren oder, falls darüber nicht mehr sichere Auskunft erlangt werden kann, wenigstens nach "echten" Vorbildern der gleichen Zeit und Kultursphäre kopiert oder neu entworfen werden. Daß Material und Arbeit dieser Ergänzungen neu sind, fällt nach der Auffassung EBHaRdTs nicht ins Gewicht. Der „historische“ Denkmalwert, den ja EBHARd wesentlich anerkennt, beruht eben nicht so sehr auf Material oder Arbeit, sondern auf der Form. Gewinnen wir aus der Betrachtung einer wiederhergestellten Burg die Überzeugung, daß alle ihre Formen den Gebräuchen und Bedürfnissen des XIII. Jh. entsprechen, ohne daß ein Detail darunter uns als anachronistisch stören würde, dann entsteht in uns das begehrte Gefühl der Befriedigung über den Anblick eines so geschaffenen Kulturbildes aus dem Mittelalter, ohne daß wir uns im geringsten an den ergänzten Teilen stoßen. Die Ideenassoziationen, durch die das Lustgefühl in diesem Falle bedingt ist, knüpfen eben an die Formen an und nicht an die wirkliche Herstellungszeit. Wäre dem nicht so, dann gäbe es ja keinen historischen Roman, der sich ohne reale $\mathrm{Z}$ wischenglieder direkt an unser $\mathrm{Be}-$ wußtsein wendet, wogegen die restaurierte Burg wenigstens greifbar und sichtbar $\mathrm{zu}$ unseren Sinnen spricht.

Die Grundsätze der Auffassung des Architekten EBhaRd von Wesen und Aufgabe der Denkmalpflege müssen somit lauten: Wir schützen die Denkmale wegen ihres historischen Wertes; und um diesen historischen Wert zur vollen Geltung zu bringen, müssen wir die Denkmale nach Bedarf wiederherstellen.

Es sind, wie man sieht, die gleichen Grundsätze, die im letzten Drittel des XIX. Jh. die öffentliche Denkmalpflege fast durchwegs beherrscht haben; in Österreich war ihr hervorragendster Vertreter FrIedrich v. Schmid. Eine unter den Gebildeten so weitverbreitete und tiefgewurzelte Neigung wie jene für historische Ideenassoziationen konnte natürlich nicht mit einem Schlage verschwinden, als gegen Ende des vorigen Jahrhunderts allmählich aus dem "historischen" Werte der
Denkmale der unmittelbar zum Gefühle sprechende "Alterswert" sich zu entwickeln begann. ${ }^{1}$ ) Auch heute noch begegnet der historische Denkmalwert häufiger und mitunter begeisterter Schätzung, und EBHARd ist daher völlig im Rechte, wenn er als stärkstes Argument zugunsten seiner Auffassung die Aufträge zitiert, die noch fortdauerrd in Deutsch land auf Wiederherstellung von Burgen erteilt werden. Eine bestimmte Wertschätzung für das "historische" Denkmal ist also zweifellos heute noch vorhanden; wir werden am Schlusse wenigstens im allgemeinen die Gebiete abzustecken versuchen, auf denen sich diese früher allmächtige Auffassung heute noch am kräftigsten und lebendigsten erhalten hat.

Daneben hat aber die neuere Auffassung, nach welcher der Denkmalwert in einer unmittelbaren Stimmungswirkung auf das Gefühl beruht, heute bereits solche Verbreitung gewonnen, daß auch EBHARDT dagegen die Augen nicht verschließen kann. Freilich eine Konzession an diese Auffassung, wie sie Deнго in seiner Definition vom Denkmal gemacht hat, wird man bei EBHARDT vergebens suchen. Daß er den „Zauber der sterbenden Ruine", den mindestens schon die Barockmaler entdeckt hatten, nicht verkennt, ist selbstverständlich; aber er läßt uns doch nicht im $Z$ weifel darüber, daß er die Ruine wesentlich für ein notwendiges Übel ansieht, das man „aus praktischen Gründen“ nur dann mit in Kauf nimmt, wenn die Mittel fehlen. Wo letztere vorhanden sind, ist die Wiederherstellung des Ursprünglichen absolut vorzuziehen, das heißt der Stimmungswert (für den die Burgruine übrigens ein $\mathrm{zu}$ absichtliches und daher relativ minder wirksames Medium bildet) ist nach EBHARDTS Auffassung höchstens ein schwaches Surrogat für den historischen Wert eines Denkmals.

Aber diese bedingte Anerkennung des Ruinen wertes ist es nicht allein, wodurch EBHARDT eine

1) Den Prozeß, mittels welchen der au unmittelbare Stimmungswirkung abzielende Alterswert des Denkmals aus dem auf gelehrter Bildung beruhenden historischen Werte, durch die vagbegrenzte Übergangstufe des „kulturhistorischen" Wertes hindurch hervorgegangen ist, habe ich darzulegen gesucht in der Schrift Der moderne Denkmalkultus (Wien BraumüI.LER 1903) 15-17. 
gewisse Rücksicht auf die wachsende Bedeutung des Stimmungswertes in der Denkmalpflege verrät. Wiewohl er die Formel "Konservieren, nicht restaurieren" ausdrücklich bekämpft, weil das beste Konservieren eben im Restaurieren läge und weil die bisherigen Versuche von Konservierung ohne Restaurierung sämtlich „künstlerisch schauderhaft" ausgefallen wären, ist doch der Hauptteil seiner Broschüre einer Aufzählung und Erörterung der Erhaltungsmaßregeln an Ruinen gewidmet. Die Grundsätze, die er hiefür aufgestellt hat, sind zwar zum überwiegenden Teile von der ausgesprochenen Tendenz auf Erhaltung des historischen Charakters diktiert, aber es sind darunter auch solche, die ein volles Verständnis für die Hauptmittel der Stimmungswirkung verraten. So z. B., indem EвHARdT den Fugenausstich $3-4 \mathrm{~cm}$ tiefer als die Vorderflucht der Steine zurückzulegen verlangt, um die für die Stimmungswirkung so köstlichen und unersetzlichen Schatten in den Fugen zu retten, - ein Vorgang, der leider nur zu häufig bei der Sicherung alter Mauern vernachlässigt wird. Dem historischen Werte wird durch solche Behandlung der Fugen eher entgegengearbeitet, da diese ursprünglich gewiß sauber ausgefüllt gewesen sind, was EBhardt selbst mit Recht betont; wenn er sie trotzdem klaffen läßt, damit sie Schatten hervorbringen, bezeigt er damit - vielleicht unwillkürlich - seine Reverenz vor dem Anblick des Alters an und für sich und dem darauf beruhenden Stimmungsgefühl. Es wird daher auch derjenige, der den Schwerpunkt des Denkmalwertes nicht mehr in seinem historischen Charakter sucht, die in der EвHARdt schen Broschüre entwickelten Grundsätze zur Erhaltung von Burgruinen mit Nutzen lesen.

So sieht sich selbst EBHARdt zu einer gewissen, wenn auch nicht offen eingestandenen Anerkennung des Alterswertes der Denkmale und damit zu einer teilyeisen Durchbrechung seiner Grundauffassung vom historischen Werte als dem einzigen Wertfaktor am Denkmal gezwungen. Aber noch nach einer anderen, entgegengesetzten Seite hin - nach der ästhetischen - ist eine solche Durchbrechung in seinen Ausführungen zu beobachten.

In bezug auf die Forderung historischer Treue und Echtheit entwickelt EBHARd eine puritani- sche Strenge. So verlangt er unter anderem, daß selbst für solche Arbeiten an Burgen, die für private Rechnung geführt werden, das Bauprogramm durch die Regierung (offenbar als Denkmalschutzbehörde) und auf deren Kosten ausgeführt werde; ferner daß Ausgrabungen auf Burgenterrain (von denen in der Regel die wichtigsten Anhaltspunkte für die Wiederherstellung zu erwarten stehen) Privaten überhaupt untersagt werden. Das sind lediglich im Interesse einer historisch möglichst treuen Wiederherstellung der ehemaligen Burgenformen geschöpfte Bestimmungen, wie sie kaum ein Gesetzgeber in ein Denkmalschutzgesetz aufzunehmen sich getraute, weil sie doch nur einer wissenschaftlich-gelehrten Liebhaberei Befriedigung schaffen sollen. Die ergänzten Mauerteile will ErHardt als solche stets ausdrücklich durch bestimmte Zeichen signiert sehen; je nach dem Grade der Treue in bezug auf die "Echtheit" der zugehörigen Teile bilden diese Zeichen eine Skala, wie sie selbst einer der von EBHARDT so verlästerten "Theoretiker" nicht spitzfindiger hätte ausklügeln können, und die mit dem, wie wir gleich hören werden, sonst vom Restaurator verlangten künstlerischen Schwung seltsam kontrastieren. Man möchte nun meinen, $\mathrm{da} \beta$ bei einer solchen Absicht auf absolute historische Treue, die doch nur durch das gewissenhafteste Studium alter Vorbilder und schriftlicher Quellennachrichten erreicht werden kann, der restaurierende Architekt als schöpferischer Künstler vollständig hinter dem Historiker zurücktreten würde. Aber gerade dagegen erhebt EвHARDT den lebhaftesten Protest. Nicht allein daß der Architekt zur Beschaffung der "Grundlagen" der Wiederherstellung der Beihilfe des Historikers nicht bedarf, spielt überhaupt der Künstler die entscheidende Rolle bei jeder Restaurierung. Eine solche sei stets in erster Linie eine Personenfrage, wird EвHARDt nicht müde zu wiederholen. Handelte es sich nun wirklich um eine nackte Kopierung des historisch Gegebenen, dann wäre es unverständlich, warum hiefür ein schöpferischer Künstler verlangt wird, während für eine solche Aufgabe doch der Techniker vollständig ausreicht, sofern ihm die historischen Vorbilder in unzweifelhafter Form geliefert werden. Daß bei den freien Restaurierungen in der Romantikerzeit die erfindenden Architekten die Hauptrolle gespielt haben, ist uns 
ohneweiters verständlich; freilich hat EBHARDT für jene Erstlinge der modernen Denkmalpflege, weil sie der historischen Treue ermangeln, nur tadelnde Worte. EBHARDT gesteht also mit seiner Forderung nach einem erfindenden Künstler zur Leitung aller Wiederherstellungsarbeiten an Burgen unwiderleglich ein, daß dabei Aufgaben für schöpferisch tätige Architekten zu lösen und nicht bloß historisch sichergestellte Vorbilder faksimilemäßig zu kopieren sind. ${ }^{1}$ ) Damit durchbricht er aber seine eigene sonstige Auffassung vom alleinigen "historischen" Wert aller Denkmale, denn das neu Erfundene ist eben kein Historisches und hat darum auch keinen Anspruch darauf, in uns ein treues Kulturbild früherer Zeiten zu erwecken. Und in der Tat wird der moderne, denkende und empfindende Beschauer jede Wiederherstellung einer mittelalterlichen Burg, mag er einzelne Details davon oder auch ihre Gesamtanlage dem in seiner Vorstellung vorhandenen Bilde einer solchen noch so entsprechend finden, mit einem unüberwindlichen Reste von Mißtrauen betrachten, weil er eben dabei den Gedanken nicht los werden kann, daß ein moderner, schöpferischer Künstler diese Verhältnisse, Linien, Formen nachempfunden und in Wirklichkeit umgesetzt hat.

Man muß also EBHARDt in der Tat darin Recht geben, daß eine absolut vollständige Wiederherstellung einer Burg nach vorhandenen gesicherten Grundlagen kaum in einem Falle möglich und dabei fast immer der allerdings "im alten Geiste" erfindenden Ergänzungstätigkeit des schöpferischen Künstlers stets mehr oder minder breiter Spielraum gewährt ist. Dann darf man aber auch nicht verlangen, daß der Beschauer die Wiederherstellung für ein treues, kulturgeschichtliches Bild der Vergangenheit nimmt; vor einigen Dezennien hatte man es damit freilich nicht so streng genommen, heute aber, nachdem unsere Sinne in bezug auf

1) In diesem Zusammenhange darf auch noch einmal auf die schon an früherer Stelle zitierte Äußerung verwiesen werden, daß die bisherigen Versuche reiner Erhaltungsmaßnahmen an Burgruinen ein "künstlerisch schauderhaftes" Ergebnis gezeitigt hätten. Darin gelangt eine Wertbeurteilung des Denkmals vom Standpunkte des modernen Kunstgeschmacks zum Ausdrucke, wie sie mit dem Denkmalbegriff im Grunde unvereinbar, bei einem schaffenden Künstler allerdings sehr begreiflich ist. das historische Detail infolge unablässiger tiefschürfender Studien unendliche Schärfung erfahren haben, ist über diesen Sachverhalt wohl keine Täuschung mehr zulässig.

Damit sind wir zu dem Punkte gelangt, aus dem das grundsätzliche Mißverständnis zwischen Kunsthistorikern und Architekten in bezug auf die Denkmalpflege entsprungen ist und, wie gerade die beiden in Rede stehenden Schriften wieder beweisen, noch heutigen Tages immer neu geboren wird. EBhardt sagt: Der Restaurator strebe nur historische Treue an, der Historiker (er nennt ihn "Theoretiker") habe aber dabei nichts zu tun. Nun wird selbst von unbefangenen Architekten zugegeben, daß alle "historische" Restaurierungstätigkeit auf einer Kooperation der beiden Berufskreise beruht, denn EвHARDT stellt sich doch das Be-schaffen der Grundlagen allzu leicht vor, wenn er meint, daß es vom schaffenden Architekten so nebenher besorgt werden könne. Da ist es nun nicht unbegreiflich, wenn ein Historiker, der ebenfalls wenigstens zum Teile an der Theorie vom "historischen" Werte des Denkmals festhält, die entgegengesetzte Meinung zum Ausdrucke bringt. "Die Denkmalpflege ist nicht eine Aufgabe für Künstler, sondern sie liegt wesentlich im Bereiche des historischen und kritischen Denkens." So äußert sich Deнio an jener Stelle seiner Schrift, wo er sich mit dem Restaurierungswesen unserer Zeit auseinandersetzt.

Es scheint nun, daß es dieser Satz ist, der die Kunsthistoriker davon zurückgehalten hat, Deнiо sofort in jenem Maße zuzustimmen, als seine Darlegungen verdient hätten. Es ist in der Tat dagegen auf das lebhafteste zu protestieren. Vor allem widerspricht Deнio damit seiner eigenen, von ihm früher proklamierten Erkenntnis, daß wir imDenkmal "ein Stück nationalen Daseins"schützen. Das Nationalgefühl als Basis des Denkmalwertes hat weder mit dem Bereich des historischen noch mit jenem des kritischen Denkens etwas zu tun. Was Deнto zu dieser seiner eigenen Grundüberzeugung widersprechenden Äußerung geführt hat, ist, wie schon früher angedeutet wurde, nichts anderes als die Vermengung des von ihm richtig erkannten Gefühlsmoments mit dem davon gänzlich verschiedenen, tatsächlich wesentlich im kritischen Bereiche gelegenen historischen Momente 
im Denkmalbegriff. DeHio empfand deutlich, daß mit diesem Begriffe in seiner modernsten Entwicklung der erfindende Künstler nichts zu schaffen habe; in der Polemik gegen diesen übersah er aber, daß auch der Historiker nicht mehr das zuständige Forum für die Beurteilung desDenkmalwertes bildet. Die Denkmalpflege ist gewiß nicht eine Aufgabe für Künstler, sie liegt aber auch nicht mehr wesentlich im Bereiche des historischen und kritischen Denkens, sondern sie ist überwiegend bereits zur Gefühlssache geworden.

Wird erst einmal diese Anschauung allgemein durchgedrungen sein, dann ist auch das Mißverständnis zwischen Architekten und Historiker in Wegfall gekommen, denn nur' das "Historische" im Denkmalbegriff bildet den Zankapfel zwischen den Parteien. Heute sind wir freilich noch nicht so weit, und Herr EBHARDT wird wohl nicht allein noch viele Burgruinen nach seinen vortrefflichen Grundsätzen erhalten, sondern auch zahlreiche Burgen mit seiner „künstlerischen Fähigkeit des Architekten" wiederherstellen. Obgleich unsere historische Detailbildung bereits so weit vorgeschritten ist, daß wir längst einzusehen gelernt haben, wie unvollkommen die Befriedigung unseres Triebes nach anschaulicher, kulturgeschicht- licher Erkenntnis ist, die uns das Stückwerk, wie es restaurierte Burgen und Kirchen, Museumssammlungsobjekte und dergleichen darbieten, zu vermitteln vermag, so hat die Freude an solchen "lebenden Bildern" aus der Geschichte doch noch immer ihre zahlreichen Anhänger. Die Objekte hiefür sind hauptsächlich entweder in fortdauerndem Gebrauche erhaltene Bauten, wie die mittelalterlichen Dome, die man nicht dem Verderben preisgeben kann und doch nicht mit modernem Flickwerk ergänzen mag, oder aber Werke, die aus ganz anderm Kulturvoraussetzungen als die modernen hervorgegangen sind und schon aus diesem Grunde keine modernen Formen in sich aufnehmen können. $\mathrm{Zu}$ der letzteren Gattung żählen aber die Burgen. Daß gerade sie, trotz des starken (allzustarken) Stimmungseindruckes, den ihre Ruinen gewähren, heute mit so viel Vorliebe der Wiederherstellung zugeführt werden, mag sich wenigstens zum Teile auch aus dem begreiflichen Wunsche ihrer adeligen Besitzer erklären, in Erinnerung an den Ursprung ihres Standes aus dem Waffenhandwerk mit den wehrhaften Burgen gewissermaßen ein Stück ihres eigenen vergangenen Daseins wenigstens in der äuferen Form wieder aufleben zu sehen.
Alois RiegL 